\title{
The effect of magnesium sulfate administration for neuroprotection on latency in women with preterm premature rupture of membranes
}

\begin{abstract}
Amanda L. Horton, MD, Yinglei Lai, PhD, Dwight J. Rouse, Catherine Y. Spong, M.D., Kenneth J. Leveno, M.D., Michael W. Varner, M.D., Brian M. Mercer, M.D., Jay D. lams, M.D. Ronald J. Wapner, M.D., Yoram Sorokin, M.D., John M. Thorp, M.D., Susan M. Ramin, M.D., Fergal D. Malone, M.D., Mary J. O'Sullivan, M.D., Gary D. V. Hankins, M.D., Steve N. Caritis, M.D., and the Eunice Kennedy Shriver National Institute of Child Health and Human Development Maternal-Fetal Medicine Units Network

Departments of Obstetrics and Gynecology at the NorthShore University HealthSystem, Evanston, IL; University of Alabama at Birmingham, Birmingham, AL; University of Texas Southwestern Medical Center, Dallas, TX; University of Utah, Salt Lake City, UT; Case Western Reserve University-MetroHealth Medical Center, Cleveland, $\mathrm{OH}$, and University of Tennessee, Memphis, TN; The Ohio State University; Thomas Jefferson University and Drexel University, Philadelphia, PA; Wayne State University, Detroit, MI; University of North Carolina at Chapel Hill, Chapel Hill, NC; University of Texas Health Science Center at Houston-Children's Memorial Hermann Hospital, Houston, TX; Columbia University, New York, NY; University of Miami, Miami, FL; Northwestern University, Chicago, IL; University of Texas Medical Branch, Galveston, TX; University of Pittsburgh, Pittsburgh, PA; and The George Washington University Biostatistics Center, Washington, DC; the National Institute of Neurological Disorders and Stroke, Bethesda, MD; and the Eunice Kennedy Shriver National Institute of Child Health and Human Development, Bethesda, MD
\end{abstract}

\section{Abstract}

Objective-To evaluate whether magnesium sulfate administration for neuroprotection prolongs latency in women with preterm premature rupture of membranes between 24 and $316 / 7$ weeks' gestation.

Study Design-This is a secondary analysis of a randomized controlled trial of magnesium sulfate for prevention of cerebral palsy. Gravid women with a singleton pregnancy between 24 and 31 6/7 weeks' gestation with preterm premature rupture of membranes (pPROM) without evidence of labor were randomized to receive magnesium sulfate, administered intravenously as a 6-g bolus followed by a constant infusion of $2 \mathrm{~g}$ per hour up to 12 hours, or placebo. Maternal outcomes for this analysis were delivery in less than 48 hours and in less than 7 days from randomization. Neonatal outcomes included a composite of respiratory distress, interventricular hemorrhage grades 3 or 4, periventricular leukomalacia, sepsis, necrotizing enterocolitis, retinopathy of prematurity, or death.

Correspondence to the manuscript: Amanda L. Horton, MD, 2650 Ridge Ave, Evanston, Illinois 60201, horton.alm@gmail.com, Phone: 847-570-4038 (work); 847-840-9047 (cell); 847-570-1910 (fax). 
Results-A total of 1259 women were included. The rate of delivery < 48 hours was not different in the magnesium sulfate and the placebo groups $(22.2 \%$ and $20.7 \%, \mathrm{p}=0.51)$. Delivery < 7 days was similar between groups $(55.4 \%$ and $51.4 \%, \mathrm{p}=0.16)$. Median latency was also similar between groups (median [interquartile range] 6.0 days [2.4-13.8] and 6.6 days [2.4-15.1], $\mathrm{p}$ $=0.29$ ). Composite neonatal outcomes did not differ between groups. Conclusion: Magnesium sulfate administration given for neuroprotection in women with a singleton gestation with preterm premature rupture of membranes and without labor before 32 weeks does not impact latency.

\section{Keywords}

latency; magnesium sulfate; neuroprotection; preterm premature rupture of membranes

\section{Introduction}

Preterm premature rupture of membranes (PPROM), defined as spontaneous rupture of membranes before 37 weeks of gestation and the onset of contractions, affects $2-3 \%$ of pregnancies in the United States ${ }^{1}$ and accounts for approximately one third of all preterm births. ${ }^{2}$ More than $90 \%$ of gravid women who experience PPROM prior to 34 weeks deliver in less than one week and PPROM is a significant cause of neonatal morbidity and mortality. ${ }^{3}$ One of the characteristic features of PPROM is the brief time from rupture of membranes to delivery, with the duration of time increasing with decreasing gestational age. Given that neonatal morbidities are strongly correlated with gestational age at delivery, strategies to extend latency for those pregnancies that may benefit from the delay in delivery are often employed. In contemporary practice, conservative management of PPROM may include antibiotic administration to prolong latency, corticosteroid use to reduce prematurity related neonatal morbidity, and tocolytic therapy. ${ }^{1,4-6}$

Preterm birth is a risk factor for cerebral palsy, a condition characterized by abnormal control of movement and posture that results in limitation of activity. Currently, one third of cases of cerebral palsy are associated with early preterm birth. ${ }^{7}$ Three major randomized clinical trials suggest that magnesium sulfate administered before an anticipated early preterm birth reduces the risks of cerebral palsy in surviving infants. ${ }^{8-10}$ In March 2010, the American College of Obstetricians and Gynecologists (ACOG) and Society for Maternal Fetal Medicine released a joint clinical opinion stating that the available evidence suggests that magnesium sulfate is a fetal neuroprotectant. These studies included gravid women with heterogeneous risk factors for preterm birth and did not demonstrate significant pregnancy prolongation when magnesium sulfate was given for neuroprotection. ${ }^{11}$

The objective of this study was to evaluate whether magnesium sulfate administration for neuroprotection increases latency, specifically in women with preterm premature rupture of membranes between 24 and 316/7 weeks' gestation.

\section{Materials and Methods}

This is a secondary analysis of the Eunice Kennedy Shriver National Institute of Child Health and Human Development Maternal-Fetal Medicine Units Network magnesium sulfate trial for the prevention of cerebral palsy (BEAM trial). That study was a randomized 
double blind placebo controlled study of 2241 women with a singleton or twin pregnancy between 24-31 6/7 weeks gestation at imminent risk for delivery. Full details on the study design, data collection, and results have previously been reported. ${ }^{10}$

Inclusion criteria for this secondary analysis included women with a singleton pregnancy with preterm premature rupture of membranes without evidence of labor between 24-31 6/7 weeks' gestation. The diagnosis of PPROM was based on documentation in the medical records and included the presence of ferning, vaginal pooling, and a positive nitrazine test. Exclusion criteria for this analysis included any of the following at the time of randomization: labor (defined as $>4 \mathrm{~cm}$ cervical dilation and $\geq 6$ contractions/hour), twin pregnancy, suspected chorioamnionitis, previous administration of magnesium sulfate, or delivery within one hour after randomization. Magnesium sulfate was administered intravenously as a 6-g bolus followed by a constant infusion of $2 \mathrm{~g}$ per hour for 12 hours if undelivered, or placebo. If delivery had not occurred after 12 hours and was no longer considered imminent, the infusion was discontinued and resumed when delivery was deemed imminent again (e.g., when contractions developed) until the gestational age had reached 34 weeks. If at least 6 hours had passed since the discontinuation of the study medication and retreatment was necessary, another 6-g loading dose was given. Retreatment was withheld in cases where preeclampsia or eclampsia developed (in which case open-label magnesium sulfate was administered for seizure prophylaxis), if it was thought that a delay in delivery in order to administer retreatment would be detrimental to the mother or the fetus, or if the gestational age was greater than 34 weeks. In a small number $(n=14)$, the infusion was increased above $2 \mathrm{~g}$ per hour and these women were excluded from the analysis cohort. The management of women with PPROM (e.g., antibiotic use) was institution dependent.

The outcomes of interest in this study were delivery within 48 hours and delivery within 7 days from randomization. Secondary outcomes included maternal outcomes and complications and neonatal outcomes. Neonatal outcomes included a composite of respiratory distress (RDS), interventricular hemorrhage (IVH grades 3 or 4), periventricular leukomalacia (PVL), sepsis, necrotizing enterocolitis (NEC), retinopathy of prematurity (ROP), or death (collected until hospital discharge).

Continuous variables were analyzed using the Wilcoxon rank sum test. Categorical variables were analyzed using the chi-square test or the Fisher's exact test where appropriate. The proportion of women in each study group remaining pregnant was compared using survival analysis with the log rank test used to assess for the difference between the survival curves. A p-value of $<0.05$ was considered significant.

\section{Results}

A total of 2241 women were enrolled in the original BEAM study; 1925 (85.9\%) presented with PPROM. After the exclusion criteria were applied, 1259 women met criteria for this secondary analysis. $621(49.3 \%)$ received magnesium and $638(50.7 \%)$ received placebo. Demographic characteristics of the cohort are shown in Table 1. As shown in Figure 1, there was no difference in the rates of delivery within 48 hours and 7 days from randomization 
(latency) in women receiving magnesium sulfate and those receiving placebo $(<48$ hours, $22.2 \%$ vs $20.7 \%, \mathrm{p}=0.51 ;<7$ days, $55.4 \%$ vs $51.4 \%, \mathrm{p}=0.16$ ). Median latency and gestational age at delivery were similar between groups (latency: median [interquartile range [6.0 days [2.4-13.8] and 6.6 days [2.4-15.1], $\mathrm{p}=0.29$; gestational age: median [interquartile range] 30.4 weeks [28.0-31.9] and 30.1 weeks [27.9-31.9], $\mathrm{p}=0.54$ ), as were the survival curves from randomization to delivery $(\mathrm{p}=0.20)$, as shown in Figure 2. Among this cohort of women, $8.3 \%$ achieved 34 weeks gestation. The use of tocolytic medications after randomization was infrequent in either group $2.1 \%$ (magnesium) vs $1.7 \%$ (placebo), $\mathrm{p}=$ 0.63. Rates of antepartum complications including placental abruption and chorioamnionitis were similar between the magnesium sulfate and placebo groups: $8.1 \%$ and $8.0 \%$, respectively $\mathrm{p}=0.97$ ), and $12.6 \%$ and $11.8 \%$, respectively $(\mathrm{p}=0.66)$. Rates of postpartum endometritis did not differ between groups (magnesium $5.8 \%$ vs. placebo $7.1 \%, p=0.36$ ). Composite neonatal outcomes did not differ between groups (Table 2). However, infants exposed to magnesium sulfate had significantly lower rates of IVH (grade 3 or 4) when compared with placebo $(0.7 \%$ vs $2.2 \%$, OR $0.31 ; 95 \%$ CI $0.10-0.96)$.

\section{Discussion}

Magnesium sulfate administration for neuroprotection in women with a singleton gestation with preterm premature rupture of membranes without labor before 32 weeks' gestation had no significant impact on rate of delivery within 48 hours or 7 days of randomization.

Antepartum maternal complications were not increased with magnesium exposure. Additionally, there were no differences in composite neonatal outcomes.

The rates of delivery within 48 hours and within 7 days from randomization are consistent with what has been reported in the literature. In a randomized double blind placebo controlled trial of ampicillin and erythromycin versus placebo in women with PPROM between 24-32 weeks, Mercer et al demonstrated rates of delivery within 48 hours and within 7 days of $27.3 \%$ and $55.5 \%$ in women who received antibiotic therapy. ${ }^{12}$ Christmas et al. performed a randomized controlled trial of ampicillin, gentamicin, and clindamycin in 94 women who experienced preterm premature rupture of membranes between 20-34 weeks. In this study, 58.3\% of women who received antibiotic therapy delivered within 7 days of randomization. ${ }^{13}$ While there is benefit to antibiotic therapy and corticosteroid administration in women who experience PPROM, our data support the preexisting literature suggesting these women remain at very high risk for delivery within 7 days.

Interestingly, maternal outcomes including chorioamnionitis and endometritis were lower in our study than previously reported by Mercer et al. ${ }^{12}$ In our study, the rate of chorioamnionitis was $12 \%$ which is nearly one half of the $23 \%$ reported in the women whom received ampicillin and erythromycin in the Mercer study. The rate of endometritis among the cohort of women in our study was $6.4 \%$, which was also lower than the $11.8 \%$ reported in the Mercer study. ${ }^{12}$

The finding of decreased rates of IVH in those infants exposed to magnesium sulfate is of interest and provides additional support to the neuroprotective effects of antenatal magnesium sulfate exposure. However, previous studies evaluating magnesium sulfate 
exposure and IVH have produced conflicting results. ${ }^{14-15}$ Mittendorf et al ${ }^{14}$ performed a randomized controlled trial of women in preterm labor to magnesium sulfate or placebo and found that infants diagnosed with IVH were born to mothers with higher serum ionized magnesium rates. These results provided speculation that magnesium sulfate at higher doses may interact with other factors that promote brain injury or may interfere with the newborn coagulation cascade and promote brain injury through platelet dysfunction. A case control study by Petrova et $\mathrm{al}^{15}$ found that in preterm infants born between 23-31 weeks' gestation diagnosed with IVH, exposure to magnesium sulfate appeared to be protective (adjOR 0.47 (95\% CI 0.24-0.91). A direct comparison between our findings and the above studies must be interpreted with caution. Magnesium sulfate was not used as a 'tocolytic' and serum ionized magnesium levels were not evaluated.

The management of women with PPROM varies among obstetric providers and has evolved over the past 20 years. Previous estimates suggested that 1 in 3 providers administered tocolytic therapy in the setting of PPROM in the 1980s. In the mid 1990s, it appeared that tocolytic use after PPROM was increasing, with nearly $75 \%$ of clinicians in the U.S. and abroad either using or would consider using tocolytic therapy. ${ }^{6}, 16-17$

In a survey of 731 Fellows and Members of the Royal College of Obstetricians and Gynecologists of Australia and New Zealand, $75 \%$ of obstetricians administered tocolytic therapy after PPROM. ${ }^{15}$ Similarly, Ramsey et al., ${ }^{6}$ in a survey of 1375 maternal fetal medicine specialists, found that tocolytic agents were used by $73 \%$ of subspecialists. Magnesium sulfate was the most commonly used tocolytic (98\%) and tocolytic was generally used for 48 hours or less to allow for the benefits of corticosteroids. Tocolytic use in our study was proscribed by the study protocol other than oral terbutaline, but its use was infrequent. Given that recent literature does not find sufficient evidence to support the use of tocolytics in women with PPROM, this may also reflect a change in provider practice patterns. $^{18}$

The limitations of our study warrant discussion. Our outcomes of delivery within 48 hours and 7 days were after randomization, not PPROM. These time frames were chosen based on previous studies of latency antibiotics in women with PPROM. Thus, given that the median gestational age from PPROM to enrollment was over 24 hours, these may not be applicable to current clinical practice, as magnesium sulfate administration is often begun shortly after admission along with the use of latency antibiotics and corticosteroid administration. As management of PPROM was institution dependent, antibiotic regimens for latency may have differed and obstetrical management, including timing of delivery was not uniform. We did not perform a post hoc power analysis, as this practice is not appropriate in the setting of a fixed sample size, such as in the case of secondary analyses.

Given that several studies have demonstrated its beneficial effects in reduction of cerebral palsy in preterm infants, the use of magnesium sulfate for neuroprotection is likely to increase and may be adopted as standard clinical practice, similar to the use of latency antibiotics and corticosteroid administration, especially in the setting of preterm premature rupture of membranes. ${ }^{8-10} \mathrm{We}$ conclude that magnesium sulfate administration for fetal 
neuroprotection does not increase the duration of latency in women with a singleton pregnancy whom experience PPROM without labor between 24-32 weeks' gestation.

\section{Acknowledgments}

The project described was supported by grants from the Eunice Kennedy Shriver National Institute of Child Health and Human Development (NICHD) [HD27869, HD34208, HD34116, HD40544, HD27915, HD34136, HD21414, HD27917, HD27860, HD40560, HD40545, HD40485, HD40500, HD27905, HD27861, HD34122, HD40512, HD53907, HD34210, HD21410, HD36801, HD19897], MO1-RR-000080, and by the National Institute of Neurological Disorders and Stroke (NINDS) and does not necessarily represent the official views of the NICHD, NINDS, or the National Institutes of Health

The authors thank the following Network members who participated in protocol development and coordination between clinical research centers (Allison T. Northen, M.S.N, R.N.), protocol/data management and statistical analysis (Steven Weiner, M.S. and Elizabeth Thom, Ph.D.), protocol development and oversight (Deborah G. Hirtz, M.D. and Alan M. Peaceman, M.D.), and manuscript drafting and review (Mara Dinsmoor, M.D.).

In addition to the authors, other members of the Eunice Kennedy Shriver National Institute of Child Health and Human Development Maternal-Fetal Medicine Units Network are as follows:

Northwestern University, Chicago, IL - A. Peaceman, M. Dinsmoor, G. Mallett, M. Ramos-Brinson., P. Simon

University of Alabama at Birmingham, Birmingham, AL - J.C. Hauth, A. Northen, T. Hill-Webb, S. Tate, K. Nelson, F. Biasini

University of Utah, Salt Lake City, UT - L. Fullmer, K. Anderson, A. Guzman, M. Jensen, L. Williams

University of Texas Southwestern Medical Center, Dallas, TX - M.L. Sherman, J. Dax, L. Faye-Randall, C. Melton, E. Flores

Case Western Reserve University-MetroHealth Medical Center, Cleveland, OH - M. Collin, G. VanBuren, C. Milluzzi, M. Fundzak, C. Santori

The Ohio State University, Columbus, $\mathrm{OH}$ - F. Johnson, M.B. Landon, C. Latimer, V. Curry, S. Meadows

Thomas Jefferson University, Philadelphia, PA - A. Sciscione, M.M. DiVito, M. Talucci, S. Desai, D. Paul

University of Tennessee, Memphis, TN - B.M. Sibai, R. Ramsey, W. Mabie, L. Kao, M. Cassie

Wayne State University, Detroit, MI - G.S. Norman, D. Driscoll, B. Steffy, M.P. Dombrowski

Wake Forest University Health Sciences, Winston-Salem, NC - P.J. Meis, M. Swain, K. Klinepeter, M. O'Shea, L. Steele

University of North Carolina at Chapel Hill, Chapel Hill - K.J. Moise, Jr., S. Brody, J. Bernhardt, K. Dorman

University of Texas Health Science Center at Houston-Children's Memorial Hermann Hospital, Houston, TXL.C. Gilstrap, III, M.C Day, E. Gildersleve, F. Ortiz, M. Kerr

Columbia University, New York, NY - V. Pemberton, L. Paley, C. Paley, S. Bousleiman, V. Carmona

Brown University, Providence, RI - M. Carpenter, J. Tillinghast, D. Allard, B. Vohr, L. Noel, K. McCarten

University of Cincinnati, Cincinnati, $\mathrm{OH}$ - M. Miodovnik, N. Elder, W. Girdler, T. Gratton

University of Chicago, Chicago, IL - A.H. Moawad, M. Lindheimer, P. Jones

University of Miami, Miami, FL - F. Doyle, C. Alfonso, M. Scott, R. Washington

University of Texas Medical Branch, Galveston, TX - T. Wen, L.A. Goodrum, G.R. Saade, G.L. Olson, H.M. Harirah, E. Martin

University of Texas at San Antonio, San Antonio, TX - O. Langer, E. Xenakis, D. Conway, M. Berkus 
University of Pittsburgh, Pittsburgh, PA - T. Kamon, M. Cotroneo, C. Milford

The George Washington University Biostatistics Center - E.A. Thom, S.J. Weiner, B. Jones-Binns, M. Cooney, M. Fischer, S. McLaughlin, K. Brunette, E. Fricks

National Institute of Neurological Disorders and Stroke, Bethesda, MD - D. Hirtz, K.B. Nelson

Eunice Kennedy Shriver National Institute of Child Health and Human Development, Bethesda, MD - S. Tolivaisa, D. McNellis, C. Catz, K. Howell

MFMU Network Steering Committee Chair (University of Pittsburgh, Pittsburgh, PA) - J. Roberts

\section{Reference}

1. Siggh K, Mercer BM. Antibiotics after preterm premature rupture of the membranes. 2011; 54:344350 .

2. Goldenberg RL, Culhane JF, Iams JD, Romero R. Epidemiology and causes of preterm birth). Lancet. 2008; 371:75-84. [PubMed: 18177778]

3. Ehrenberg HM, Mercer BM. Antibiotics and the management of preterm premature rupture of membranes. Clin Perinatol. 2001; 28:807-818. [PubMed: 11817191]

4. Vidaeff AC, Ramin SM. Antenatal corticosteroids after preterm premature rupture of membranes. Clin Obstet Gynecol. 2011; 54:337-343. [PubMed: 21508704]

5. ACOG Committee on Obstetric Practice. ACOG Committee Opinion No 475. Antenatal corticosteroid therapy for fetal maturation. Obstet Gynecol. 2011; 117:422-424. [PubMed: 21252775]

6. Ramsey PS, Nuthalapaty FS, Lu G, Ramin S, Nuthalapaty ES, Ramin KD. Contemporary management of preterm premature rupture of membranes (PPROM): a survey of maternal fetal medicine providers. Am J Obstet Gynecol. 2004; 191:1497-1502. [PubMed: 15507990]

7. Winter S, Autry A, Boyle C, Yeargin, Allsopp M. Trends in the prevalence of cerebral palsy in a population based study. Pediatrics. 2006; 118(6):e1621-e1626. [PubMed: 17074842]

8. Crowther CA, Hiller JE, Doyle LW, Haslan RR. Effect of magnesium sulphate given for neuroprotection before preterm birth: a randomized controlled trial. JAMA. 2003; 290:2669-2676. [PubMed: 14645308]

9. Marret S, Marpeau L, Zupan-Simunek V, Eurin D, et al. Magnesium sulphate given before verypreterm birth to protect infant brain: the randomised controlled PREMAG trial. PREMAG trial group. BJOG. 2007

10. Rouse DJ, Hirtz DG, Thom E, et al. A randomized, controlled trial of magnesium sulfate for the prevention of cerebral palsy. N Engl J Med. 2008; 395:895-905. [PubMed: 18753646]

11. Magnesium sulfate before anticipated preterm birth for neuroprotection. Committee Opinion No. 455. American College of Obstetricians and Gynecologists. Obstet Gynecol. 2010; 115:669-671. [PubMed: 20177305]

12. Mercer BM, Miodovnik M, Thurnau GR, et al. Antibiotic therapy for reduction of infant morbidity after preterm premature rupture of membranes. JAMA. 1997; 278:989-995. [PubMed: 9307346]

13. Christmas JT, Cox SM, Andres W, Dax J, Leveno KJ, Gilstrap LC. Expectant management of preterm ruptured membranes: effect of antimicrobial therapy. Obstet Gynecol. 1992; 80:759-762. [PubMed: 1407911]

14. Mittendorf R, Dambrosia J, Dannmann O, et al. Association between maternal serum ionized magnesium levels at delivery and neonatal ventricular hemorrhage. J Pediatr. 2002; 140:540-546. [PubMed: 12032519]

15. Petrova A, Mehta R. Magnesium sulfate tocolysis and intraventricular hemorrhage in very preterm infants. Indian J Pediatr. 2012; 79:43-47. [PubMed: 21625843]

16. Capeless EL, Mead PB. Management of preterm premature rupture of membranes: a lack of national consensus. Am J Obstet Gynecol. 1987; 157:11-12. [PubMed: 3605242]

17. Buchanan S, Crowther C, Morris J. Preterm prelabour rupture of membranes: a survey of current practice. Aust NZ J Obstet Gynaecol. 2004; 44:400-403. 
18. Mackeen AD, Seibel-Seamon J, Grimes-Dennis J, Baxter JK, Berghella V. Tocolytics for preterm premature rupture of membranes. Cochrane Database Systm Rev. 2011; 10:CD007062. 


\section{Clinical Perspective}

Magnesium sulfate administration for neuroprotection does not prolong latency in women with preterm premature rupture of membranes between 24 and 31 6/7 weeks' gestation. 


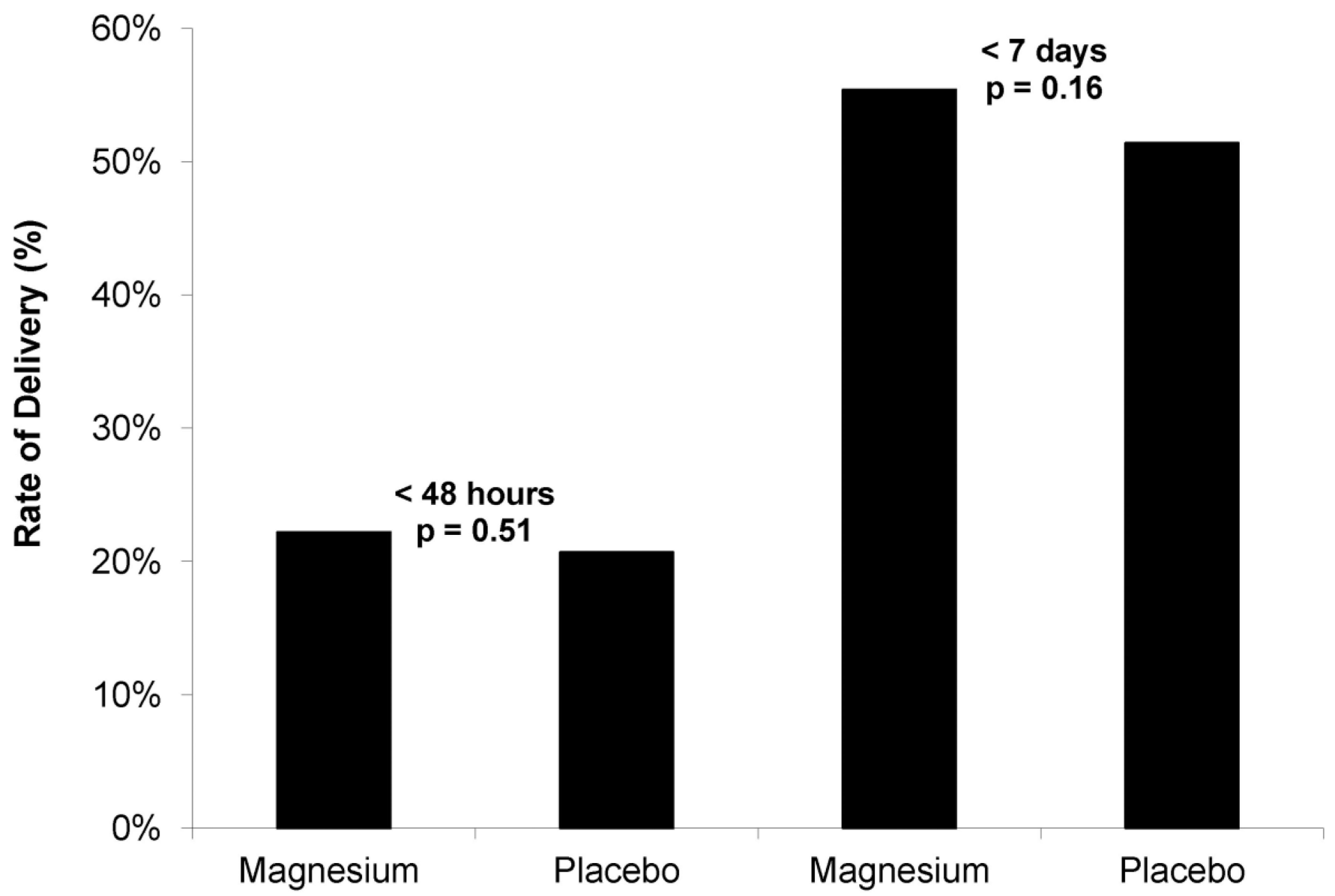

Figure 1.

Rates of delivery within 48 hours and 7 days from randomization among study group 


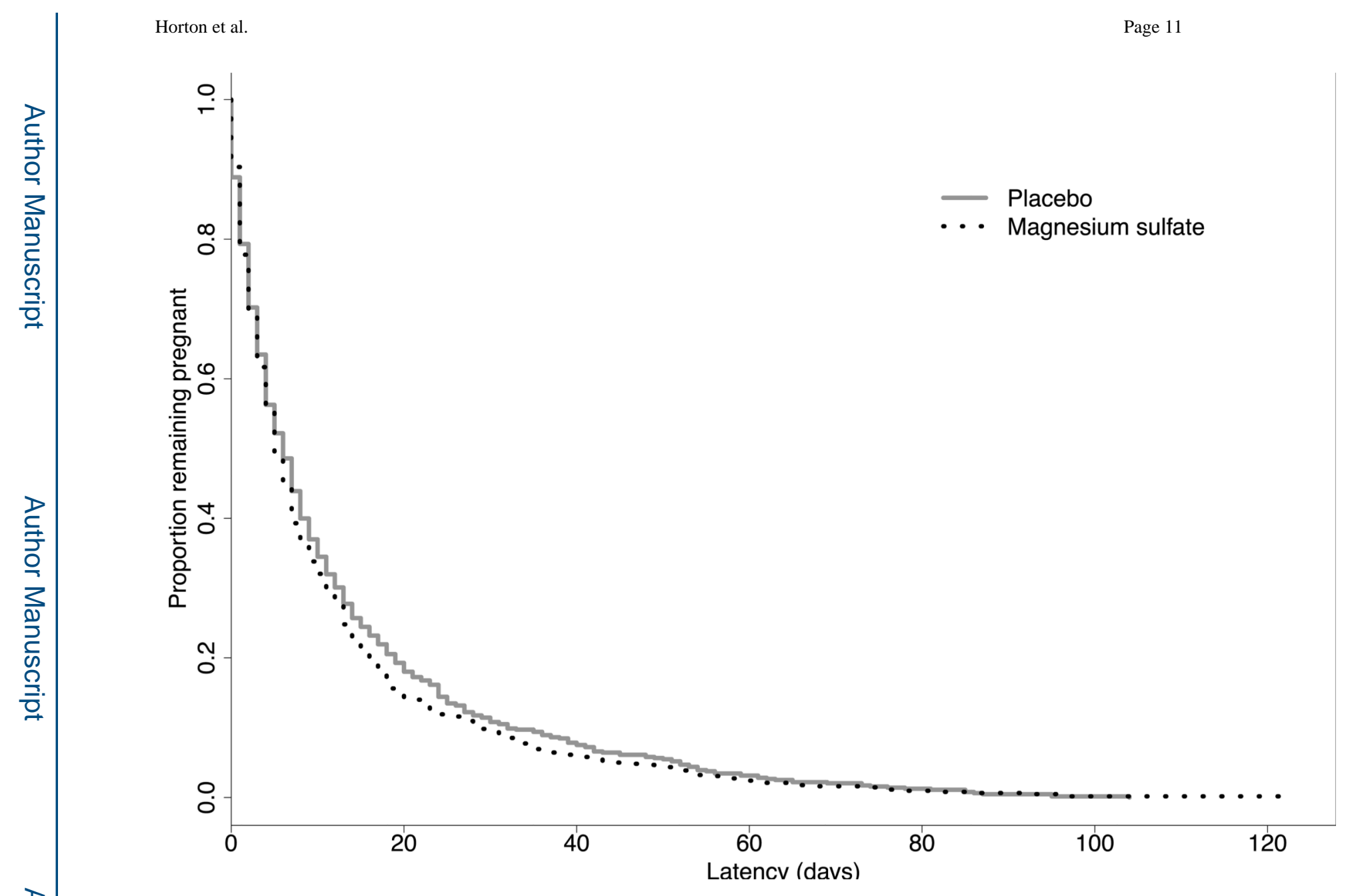

Figure 2.

Survival curves illustrating proportion of women remaining pregnant after randomization. 
Table 1

Maternal Demographic and Obstetrical Characteristics of Study Populations*

\begin{tabular}{|l|l|l|l|}
\hline & $\begin{array}{l}\text { Magnesium sulfate } \\
\text { (N=621) }\end{array}$ & $\begin{array}{l}\text { Placebo } \\
\text { (N= 638) }\end{array}$ & P value $^{\mathbf{b}}$ \\
\hline Gestational age at randomization (weeks) & $28.5 \pm 2.3$ & $28.3 \pm 2.3$ & 0.10 \\
\hline $\begin{array}{l}\text { Time from rupture of membranes to randomization (hours) } \\
\text { Median } \\
\text { [Interquartile range] }\end{array}$ & $25.3[11.3,57.0]$ & $24.9[11.7,56.9]$ & 0.92 \\
\hline Maternal age (years) & $26.8 \pm 6.4$ & $26.1 \pm 6.2$ & 0.06 \\
\hline Maternal prepregnancy body mass index & $26.3 \pm 6.7$ & $26.6 \pm 6.8$ & 0.32 \\
\hline $\begin{array}{l}\text { Race } \\
\text { Black }\end{array}$ & $275(44.3 \%)$ & $286(44.8 \%)$ & 0.98 \\
$\begin{array}{l}\text { Hispanic } \\
\text { Other }\end{array}$ & $238(38.3 \%)$ & $238(36.3 \%)$ & \\
\hline Married (no/total number) (\%) & $16(2.6 \%)$ & $16(2.5 \%)$ & \\
\hline Educational level (years) & $324 / 620(52.3 \%)$ & $283 / 636(44.5 \%)$ & 0.006 \\
\hline Nulliparous (\%) & $11.9 \pm 2.4$ & $11.9 \pm 2.4$ & 0.77 \\
\hline Smoking during pregnancy (\%) & $195 / 621(31.4 \%)$ & $213 / 638(33.4 \%)$ & 0.45 \\
\hline Prior preterm delivery & $186 / 621(30.0 \%)$ & $182 / 638(28.5 \%)$ & 0.58 \\
\hline Antenatal corticosteroid administration & $183 / 621(29.5 \%)$ & $187 / 638(29.3 \%)$ & 0.95 \\
\hline
\end{tabular}

IQR interquartile range; SD standard deviation

* Data presented as n (\%) based on 1259 women at enrollment

${ }^{\dagger} \chi^{2}$, Fisher's exact, or Wilcoxon rank sum test.

The body mass index is the weight in kilograms divided by the square of the height in meters. Values were available for 558 mothers in the magnesium sulfate group and 578 in the placebo group. 
Table 2

Neonatal Outcome According to Treatment Group*

\begin{tabular}{|l|l|l|l|}
\hline & $\begin{array}{l}\text { Magnesium sulfate } \\
(\mathbf{N = 6 2 1})\end{array}$ & $\begin{array}{l}\text { Placebo } \\
(\mathbf{N = 6 3 8})\end{array}$ & $\begin{array}{l}\text { Odds Ratio } \\
(\mathbf{9 5 \%} \text { CI })\end{array}$ \\
\hline Birthweight (grams) Mean (SD) & $1468 \pm 542$ & $1489 \pm 583$ & $\mathrm{p}=0.76^{+}$ \\
\hline 5 min Apgar < & $90 / 621(14.5 \%)$ & $103 / 636(16.2 \%)$ & $0.88(0.65-1.19)$ \\
\hline Composite outcome ${ }^{\dagger}$ & $339 / 605(56.0 \%)$ & $350 / 623(56.2 \%)$ & $0.99(0.79-1.25)$ \\
\hline Respiratory Distress Syndrome & $285 / 620(46.0 \%)$ & $293 / 633(46.3 \%)$ & $0.99(0.79-1.23)$ \\
\hline Periventricular Leukomalacia & $8 / 589(1.4 \%)$ & $14 / 602(2.3 \%)$ & $0.58(0.24-1.39)$ \\
\hline Interventricular Hemorrhage (Grade 3 or 4) & $4 / 589(0.7 \%)$ & $13 / 602(2.2 \%)$ & $0.31(0.10-0.96)$ \\
\hline Culture proven sepsis & $83 / 620(13.4 \%)$ & $88 / 633(13.9 \%)$ & $0.96(0.69-1.32)$ \\
\hline Necrotizing enterocolitis & $50 / 620(8.1 \%)$ & $49 / 633(7.7 \%)$ & $1.05(0.69-1.58)$ \\
\hline Retinopathy of prematurity & $109 / 620(17.6 \%)$ & $113 / 633(17.9 \%)$ & $0.98(0.73-1.31)$ \\
\hline Death & $27 / 621(4.4 \%)$ & $31 / 638(4.9)$ & $0.89(0.52-1.51)$ \\
\hline
\end{tabular}

SD standard deviation

*

Data presented as n (\%) based on 1259 women at enrollment

${ }^{\dagger}$ Wilcoxon rank sum test

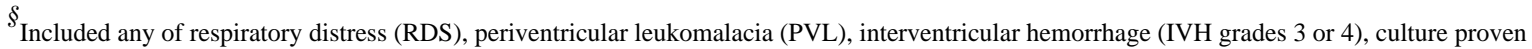
sepsis, necrotizing enterocolitis (NEC), retinopathy of prematurity (ROP), or death (collected until hospital discharge) 